\title{
Genomic knowledge in the context of diagnostic exome sequencing: changes over time, persistent subgroup differences, and associations with psychological sequencing outcomes
}

\author{
Christine Rini, $\mathrm{PhD}{ }^{1}{ }^{1}$, Gail E. Henderson, $\mathrm{PhD}^{2,3}$, James $\mathrm{P}$. Evans, $\mathrm{MD}, \mathrm{PhD}^{2,4,5}$, \\ Jonathan S. Berg, MD, PhD ${ }^{2,4}$, Ann Katherine M. Foreman, MS, CGC (iD ${ }^{2,4}$, Ida Griesemer, MSPH(10) ${ }^{6}$, \\ Margaret Waltz, PhD (iD ${ }^{2,3}$, Julianne M. O'Daniel, MS, CGC (iD ${ }^{2,4}$ and Myra I. Roche, MS, CGC (i) ${ }^{2,4,7^{\prime}}$
}

Purpose: People undergoing diagnostic genome-scale sequencing are expected to have better psychological outcomes when they can incorporate and act on accurate, relevant knowledge that supports informed decision making.

Methods: This longitudinal study used data from the North Carolina Clinical Genomic Evaluation by NextGen Exome Sequencing Study (NCGENES) of diagnostic exome sequencing to evaluate associations between factual genomic knowledge (measured with the University of North Carolina Genomic Knowledge Scale at three assessments from baseline to after return of results) and sequencing outcomes that reflected participants' perceived understanding of the study and sequencing, regret for joining the study, and responses to learning sequencing results. It also investigated differences in genomic knowledge associated with subgroups differing in race/ethnicity, income, education, health literacy, English proficiency, and prior genetic testing.

Results: Multivariate models revealed higher genomic knowledge at baseline for non-Hispanic Whites and those with higher income, education, and health literacy ( $p$ values $<0.001$ ). These subgroup differences persisted across study assessments despite a general increase in knowledge among all groups. Greater baseline genomic knowledge was associated with lower test-related distress $(p=$ $0.047)$ and greater perceived understanding of diagnostic genomic sequencing ( $p$ values 0.04 to $<0.001$ )

Conclusion: Findings extend understanding of the role of genomic knowledge in psychological outcomes of diagnostic exome sequencing, providing guidance for additional research and interventions.

Genetics in Medicine (2020) 22:60-68; https://doi.org/10.1038/s41436019-0600-4

Keywords: diagnostic exome sequencing; knowledge; decision making; disparities; genomic

\section{INTRODUCTION}

Knowledge is theorized to be central to informed patient decision making and consent in medical care and research. ${ }^{1-4}$ Accordingly, providing accurate, relevant information is critical for interventions that promote informed decisions about joining studies, adopting healthy lifestyle behaviors, and preventing or managing health problems. ${ }^{1,5,6}$ These interventions sometimes also seek to help people make valueconcordant decisions, ${ }^{1}$ underscoring that knowledge is necessary but not sufficient for informed decision making. Nonetheless, the centrality of knowledge in theoretical models of informed decision making is clear, and it reflects both the ethical need to ensure people are adequately informed about consequences of health decisions and the belief that informed choices lead to better decision outcomes. ${ }^{6}$

People undergoing diagnostic genome-scale sequencing (e.g., exome sequencing) may receive education and counseling to inform them about sequencing procedures and the information they can yield. They may also learn about principles of inheritance to clarify how sequencing results may be relevant to family members. Given the theorized importance of knowledge of these and related topics in patient decision making, we sought to examine its variation in people undergoing diagnostic exome sequencing and its prospective associations with downstream psychological outcomes including test-related distress and uncertainty, decision regret, and ${ }^{1}$ John Theurer Cancer Center, Hackensack University Medical Center, Hackensack, NJ, USA; ${ }^{2}$ Center for Genomics and Society, University of North Carolina, Chapel Hill, NC, USA;
${ }^{3}$ Department of Social Medicine, University of North Carolina at Chapel Hill, Chapel Hill, NC, USA; ${ }^{4}$ Department of Genetics, University of North Carolina at Chapel Hill, Chapel
Hill, NC, USA; ${ }^{5}$ Department of Medicine, University of North Carolina at Chapel Hill, Chapel Hill, NC, USA; ${ }^{6}$ Department of Health Behavior, University of North Carolina at
Chapel Hill, Chapel Hill, NC, USA; ${ }^{7}$ Department of Pediatrics, University of North Carolina, Chapel Hill, NC, USA. Correspondence: Christine Rini (christine.rini@northwestern.edu) 
perceived understanding. Findings could guide interventions to promote better psychological sequencing outcomes in diverse populations-an important goal given increasing use of large-scale sequencing for diagnostic purposes and the fact that the technique and spectrum of results it can provide remain relatively unfamiliar to most patients.

Patients or their proxies (e.g., parents of pediatric patients) are typically expected to take an active role in decisions about diagnostic sequencing and to collaborate with clinicians to make sound decisions about applying their results to subsequent health decisions for themselves and their family. Theoretically, these activities should lead to positive psychological sequencing outcomes (e.g., lower test-related distress and decision regret) for individuals with good foundational knowledge of genes, their health implications, how genes are inherited in families, and the strengths and weaknesses of the specific testing modality. Yet, for various reasons, many people do not have sufficient knowledge to tackle these challenges. $^{7,8}$

Challenges such as these motivated the North Carolina Clinical Genomic Evaluation by NextGen Exome Sequencing Study (NCGENES), part of the Clinical Sequencing Exploratory Research (CSER) research consortium. NCGENES investigated use of exome sequencing as a diagnostic tool and helped inform best practices for disclosing diagnostic and secondary genomic information in clinical settings. ${ }^{9,10}$ Adult and pediatric patients participating in NCGENES had diagnostic exome sequencing to elucidate the genetic cause of symptoms or health conditions that appeared to have a genetic etiology. They each received diagnostic sequencing results indicating either that no cause had been found (negative), a possible cause had been found (uncertain), or a definitive cause had been found (positive).

As part of NCGENES, we developed and validated the University of North Carolina Genomic Knowledge Scale (UNC-GKS) to assess knowledge domains thought to be critical for making informed decisions about undergoing sequencing, comprehending the meaning and limitations of results, and taking appropriate actions upon learning results. $^{11,12}$ These domains include the nature of genes, their effects on health, how genes are inherited in families, and potential benefits, harms, and limitations of exome sequencing. Put another way, the measure's items cover aspects of knowledge consistent with Smerecnik's categories: awareness knowledge (knowing that genetic risk factors exist), how-to knowledge (knowing how they influence risk for health problems), and, to a lesser extent, principles knowledge (theoretical knowledge, including knowledge concerning patterns of inheritance and disease development). ${ }^{13}$ Our research shows that the measure has sound psychometric properties across subgroups differing in sex, race/ethnicity, education, and English proficiency, and higher genomic knowledge is associated with higher health literacy and numeracy, ${ }^{14}$ consistent with the broader research literature. ${ }^{8,15}$

Little research has investigated the assumption that greater knowledge promotes salutary psychological outcomes. A few studies in oncology ${ }^{5,16}$ suggest that increases in or higher levels of relevant knowledge are associated with better outcomes. We know of no studies extending this evidence to diagnostic genome-scale sequencing. Although greater genomic knowledge could be associated with better psychological sequencing outcomes (e.g., because it reduces regret, uncertainty, and corresponding distress), it is also possible that genomic knowledge is relatively unimportant to such outcomes. For instance, they may be more related to specific, personalized interpretations and advice provided by clinicians. Accordingly, we examined associations between general genomic knowledge and the following psychological outcomes: perceived understanding of the NCGENES study and diagnostic sequencing, feelings of regret for joining the study to obtain diagnostic sequencing for themselves or their child, and responses to receiving sequencing results (testrelated distress, test-related uncertainty).

We also evaluated whether genomic knowledge varies according to social resources that may be related to greater ability to acquire, comprehend, and retain genomic knowledge. These resources include past experience with prior genetic testing ${ }^{16}$ and social contextual factors, including formal education, ${ }^{17}$ health literacy, ${ }^{15}$ and English proficiency. ${ }^{18}$ Additionally, race/ethnicity and income are likely to be proxies for a broader set of resources and experiences related to knowledge acquisition, comprehension, and retention. Consistent with this assertion, evidence shows that genetic/genomic knowledge and awareness differ by income $^{19-21}$ and race/ethnicity. ${ }^{19,22-25}$

\section{Study overview}

The present study included NCGENES participants who completed the UNC-GKS prior to enrollment (baseline), two weeks after enrollment (time 1), and two weeks after receiving positive, uncertain, or negative diagnostic sequencing results (time 2). At each timepoint, we provided education about diagnostic sequencing (e.g., through mailed brochures and discussions with study geneticists and genetic counselors). Analyses evaluated the hypotheses that (1) genomic knowledge would increase across assessments as participants received additional education; (2) baseline genomic knowledge would differ for groups differing in race/ethnicity, health literacy, income, education, English proficiency, and prior genetic testing; and (3) higher levels of baseline genomic knowledge and greater increases in genomic knowledge would be prospectively associated with better psychological sequencing outcomes. We also explored whether subgroups differed in change in genomic knowledge across the study.

\section{MATERIALS AND METHODS}

\section{Participants}

Patients were referred to NCGENES from hospitals associated with the University of North Carolina at Chapel Hill (UNC) and Vidant Medical Center (Greenville, NC). All had symptoms of an undiagnosed condition with a possible genetic etiology that had not been previously identified. 
Eligible patients had diverse indications for sequencing (e.g., neurodegenerative disorders, cardiovascular diseases, dysmorphology, hereditary cancer; see ref. ${ }^{9}$ ). The present study's sample included 315 adult patients and parents or legal guardians of pediatric patients ("caregivers") who completed the NCGENES baseline, time 1, and time 2 assessments. All were enrolled between August 2012 and December 2014 and completed study measures in English.

\section{Procedures}

Potential participants were mailed a consent form, a baseline questionnaire, and an educational brochure describing diagnostic exome sequencing and possible diagnostic results (i.e., positive, uncertain, or negative, indicating identification of a clear, possible or no genetic cause for their condition). The baseline genomic knowledge measure was in the baseline questionnaire, so some participants may have completed it after reading the brochure. We did not record whether this occurred. Participants then met with a genetic counselor. In this meeting, all participants provided who obtained signed consent from all participants, answered questions, and reiterated and extended information in the brochure, providing personalized information. Participants then returned their completed baseline questionnaire, completed a health literacy assessment, and had blood drawn for sequencing. Two weeks later, they completed the time 1 structured phone interview and mailed questionnaire.

Diagnostic sequencing results and, if found, medically actionable secondary findings were available 6-12 months later, triggering scheduling of an in-person return of results meeting. At this time, cognitively intact adult patients were randomized to one of two groups: control (received only diagnostic sequencing results) or decision (received diagnostic results, then learned about and were able to request non-medically actionable secondary sequencing findings $\left.{ }^{10,26}\right)$. The $3.4 \%$ of adult patients who received medically actionable secondary findings along with their diagnostic results were ineligible for randomization to avoid overwhelming them (e.g., due to the stress of receiving unanticipated medical information and the potential need to take medical action because of it). Caregivers were ineligible for randomization because we limited return of non-medically actionable secondary findings to consenting adults. We mailed all participants a letter confirming their return of results appointment. For the decision group, this mailing included an educational brochure describing six categories of non-medically actionable secondary findings, potential benefits and harms of learning each category, and a values clarification exercise to support informed decision making regarding requesting these findings. Because the decision group received additional education that could affect genomic knowledge, we evaluated the need to control for randomized assignment in multivariate analyses.

At their return of results visit, participants met with a study medical geneticist and genetic counselor to learn their diagnostic findings and receive counseling about these findings. Decision group participants also learned about requesting and learning non-medically actionable secondary findings. Two weeks later (before any requested non-medically actionable secondary findings were returned), all participants completed the time 2 structured phone interview and mailed questionnaire. Procedures were approved by the institutional review boards of the University of North Carolina at Chapel Hill and Vidant Medical System.

\section{Measures}

Genomic knowledge was assessed with the UNC-GKS, ${ }^{14}$ which includes 25 statements about genes, genetic effects on health, familial inheritance, and diagnostic exome sequencing. Respondents mark each as true, false, or not sure/don't know (scored as incorrect). Correct responses are scored as 1 and summed. Possible scores range from 0 to 25 .

Sociodemographic characteristics were self-reported and included age, gender, race/ethnicity, marital/partner status, educational attainment, and annual household income.

Medical characteristics (e.g., indication for sequencing) were abstracted from clinical records and confirmed at enrollment. Participants self-reported whether they (or, for caregivers, their child) had genetic testing in the past ( $1=$ yes, $0=$ no).

Health literacy was assessed by staff at enrollment using the 66-item Rapid Estimate of Adult Literacy Measure. ${ }^{27}$ As recommended, ${ }^{27}$ we summed the number of words pronounced correctly. Participants were categorized as low health literacy (scores of $0-44, \leq$ sixth grade reading level), marginal literacy (45-60, seventh to eighth grade reading level), or functional health literacy $(61-66, \geq$ ninth grade reading level). ${ }^{27}$ Because few participants (1.3\%) had low health literacy, we combined the low and marginal health literacy categories.

English proficiency in speaking, reading, and writing was assessed using a 3-item subscale of the Cultural Identity Scale ${ }^{28}$ to assess English proficiency in speaking, reading, and writing. Responses ranged from 1 (poor) to 4 (excellent) and were summed (Cronbach's $a=0.90$ ). Because most participants (85.1\%) reported excellent proficiency, we dichotomized scores to indicate high proficiency $(=12)$ and lower proficiency $(<12)$.

Test-related distress and uncertainty were assessed at time 2 with an adapted version of the Multidimensional Impact of Cancer Risk Assessment (MICRA). ${ }^{29}$ Participants reported how often in the past week they had experienced distress (6 items, e.g., anxious or nervous) and uncertainty (12 items, e.g., frustration about lack of clear guidelines for using test results) as a result of their/their child's test results. Items were reworded to accommodate the interview format, to refer to exome sequencing rather than genetic testing, and, when necessary, to account for differences between diagnostic exome sequencing and genetic tests for risk assessment (the scale's original purpose). Possible responses were: $0=$ never, $1=$ rarely, $3=$ sometimes, and $5=$ often. Internal reliability was good (Cronbach's $\mathrm{a}=0.86$ for distress, 0.88 for uncertainty). 
Decision regret was assessed with the 5-item Decision Regret Scale. Regarding their decision to have diagnostic sequencing, participants indicated their agreement with five statements (e.g., "it was the right decision") on a scale from 1 (strongly disagree) to 5 (strongly agree). Positively worded items were reverse-coded and responses were averaged $(a=$ 0.89).

Perceived understanding of diagnostic exome sequencing was assessed with six items created for this study (Online Supplemental Table 1). Responses ranged from 1 (strongly disagree) to 5 (strongly agree). Positively worded items were reverse-coded and responses were averaged $(a=0.85)$.

\section{Data analysis}

First, we generated descriptive statistics and examined the psychometric properties of measures. Because there were few missing values, they were mean or mode replaced. We generated descriptive statistics for baseline knowledge and changes in genomic knowledge across assessments (baseline, time 1, and time 2), both collapsed across subgroups and for subgroups differing in race/ethnicity, health literacy (functional versus lower), annual household income, educational attainment (high school or less versus more education), prior genetic testing (yes/no), and English proficiency (high versus lower). We conducted repeated measures analyses of covariance to evaluate subgroup differences in baseline genomic knowledge and change in genomic knowledge in a multivariate model that adjusted for covariates. Potential covariates evaluated for inclusion were study role (adult versus caregiver), participant gender, diagnostic sequencing result (positive, uncertain, or negative, with negative as the reference group), and assignment to the decision or control group. Age was not considered because it was missing for $29 \%$ of caregivers. Covariates were entered into the model if bivariate analyses showed they were associated with genomic knowledge at any timepoint.

Finally, we evaluated associations between genomic knowledge variables and test-related distress, test-related uncertainty, decision regret, and perceived understanding of diagnostic exome sequencing. Test-related distress, testrelated uncertainty, and decision regret each had two missing values, and perceived understanding had six. Analyses conducted with and without mean imputed values did not differ, so we report findings from analyses without mean imputed values. First, we examined bivariate associations between each outcome and potential covariates to identify which covariates were associated with each outcome. Next, we conducted a hierarchical linear multiple regression analysis predicting each outcome. In step 1 we entered covariates. In step 2 we entered baseline genomic knowledge and change in genomic knowledge across the three study time points.

\section{RESULTS}

Descriptive statistics are shown in Table $\mathbf{1}$ and correct responses to the genomic knowledge scale items at each assessment are shown in online Supplemental Table 2.

\section{Description of observed genomic knowledge}

Genomic knowledge scores averaged $18.72(\mathrm{SD}=5.21)$ at baseline, $20.01(\mathrm{SD}=4.53)$ at time 1 , and $20.29(\mathrm{SD}=4.25)$ at time 2. Paired samples $t$ tests indicated that these scores increased from baseline to time $1, t(314)=6.73, p<0.001$, and from baseline to time $2, t(314)=7.68, p<0.001$. They did not increase from time 1 to time $2, t(314)=1.63, p=0.11$. The standardized effect size for baseline to time 2 change was 0.44 ( $95 \%$ confidence interval 0.248-0.563), calculated using a pooled standard deviation and the correlation of 0.722 between the scores. ${ }^{30}$

\section{Subgroup differences in genomic knowledge}

One-way analyses of variance evaluating associations between social resource variables and knowledge revealed that baseline genomic knowledge was higher for non-Hispanic White participants (versus another race/ethnicity), participants with experience with prior genetic testing (versus those without this experience), and those with higher health literacy, income, and English proficiency (all $p$ values < 0.001) (Online Supplemental Table 3).

With respect to potential covariates, we found no association between genomic knowledge at any timepoint and study role ( $p \quad$ values $=0.73-0.91)$; participant gender $(p \quad$ values $=$ $0.18-0.73)$, diagnostic sequencing result $(p$ values $=$ $0.18-0.95)$, or being randomized to the decision group ( $p$ values $=0.60-0.95)$. Consequently, we did not include any covariates in the repeated measures analysis of variance predicting genomic knowledge. Model estimates therefore indicated the effect of each social resource variable on knowledge, controlling for all other social resource variables in the model. Findings revealed differences in baseline genomic knowledge for race/ethnicity $\left(p<0.001\right.$, partial $\left.\eta^{2}=0.076\right)$, income $\left(p=0.04\right.$, partial $\left.\eta^{2}=0.013\right)$, education $(p<0.001$, partial $\left.\eta^{2}=0.111\right)$, and health literacy $\left(p<0.001\right.$, partial $\eta^{2}=$ 0.095). Estimated marginal means (adjusted for other variables in the model) showed that these differences were consistent with the bivariate analyses and, in general, supported our hypothesis that baseline genomic knowledge would differ for specified groups. However, differences in baseline genomic knowledge observed in bivariate analyses were no longer observed for English proficiency $\left(p=0.08\right.$, partial $\left.\eta^{2}=0.010\right)$ or prior genetic testing $\left(p=0.13\right.$, partial $\left.\eta^{2}=0.008\right)$.

Within-subjects effects with Huynh-Feldt correction revealed an increase in knowledge scores across assessments $\left(p<0.001\right.$, partial $\left.\eta^{2}=0.063\right)$, supporting hypothesis 1 . This increase was not moderated by participant race/ethnicity, health literacy, income, education, prior genetic testing, or English proficiency ( $p$ values $=0.12-0.76$ ). Thus, for these variables, differences that did or did not appear at baseline remained stable across assessments. Figure $\mathbf{1}$ illustrates this effect using race/ethnicity as an example.

\section{Implications for psychological sequencing outcomes}

We evaluated implications of baseline genomic knowledge and change in genomic knowledge for the four outcomes, all measured at time 2. Baseline knowledge was inversely 
Table 1 Sample descriptive statistics $(N=315)$

\begin{tabular}{|c|c|c|c|c|c|}
\hline \multirow[b]{2}{*}{ Variables } & \multicolumn{2}{|c|}{ Adult patients $(N=199)$} & \multicolumn{2}{|c|}{ Caregiver participants $(N=116)$} & \multirow[b]{2}{*}{$p$ value } \\
\hline & M (SD) & $N(\%)$ & $\mathrm{M}(\mathrm{SD})$ & $N(\%)$ & \\
\hline Age & $50.88(14.67)$ & & $39.76(9.94)^{a}$ & & $<0.001$ \\
\hline \multicolumn{6}{|l|}{ Race/ethnicity } \\
\hline White, non-Hispanic & & $168(84.4 \%)$ & & $93(80.2 \%)$ & 0.31 \\
\hline Other race/ethnicity & & $31(15.6 \%)$ & & $23(19.8 \%)$ & \\
\hline Asian & & $2(1.0 \%)$ & & $0(0.0 \%)$ & \\
\hline Latinx/Hispanic & & $8(4.0 \%)$ & & $10(8.6 \%)$ & \\
\hline Other or mixed & & $5(2.5 \%)$ & & $4(3.4 \%)$ & \\
\hline Annual household income & & & & & 0.38 \\
\hline$<\$ 29,999$ & & $44(22.1 \%)$ & & $32(27.6 \%)$ & \\
\hline Missing/unreported & & $13(6.5 \%)$ & & $5(4.3 \%)$ & \\
\hline Education & & & & & 0.31 \\
\hline High school or less & & $26(13.1 \%)$ & & $19(16.4 \%)$ & \\
\hline More than high school & & $172(86.4 \%)$ & & $97(83.6 \%)$ & \\
\hline Missing & & $1(0.5 \%)$ & & $0(0 \%)$ & \\
\hline English proficiency & & & & & 0.12 \\
\hline High & & $164(82.4 \%)$ & & $104(89.7 \%)$ & \\
\hline Lower & & $35(17.6 \%)$ & & $12(10.3 \%)$ & \\
\hline Health literacy & & & & & 0.31 \\
\hline Low/marginal & & $20(10.1 \%)$ & & $7(6.0 \%)$ & \\
\hline Negative & & $132(66.3 \%)$ & & $65(72.5 \%)$ & 0.06 \\
\hline Uncertain & & $37(18.6 \%)$ & & $30(24.7 \%)$ & 0.17 \\
\hline Positive & & $30(15.1 \%)$ & & $21(18.8 \%)$ & 0.46 \\
\hline \multicolumn{6}{|l|}{ Randomization } \\
\hline Decision group & & $89(44.7 \%)$ & & $0(0 \%)$ & \\
\hline Control group & & $103(51.7 \%)$ & & $0(0 \%)$ & \\
\hline Ineligible for randomization ${ }^{b}$ & & $7(3.5 \%)$ & & $116(100 \%)$ & \\
\hline \multicolumn{6}{|l|}{ Genomic knowledge } \\
\hline Baseline & & $18.65(5.49)$ & & $18.83(4.71)$ & 0.78 \\
\hline Time 1 & & $19.94(4.69)$ & & $20.13(4.25)$ & 0.73 \\
\hline Time 2 & & $20.27(4.23)$ & & $20.33(4.30)$ & 0.91 \\
\hline
\end{tabular}

correlated with test-related distress $(r=-0.11, p=0.04)$, testrelated uncertainty $(r=-0.18, p=0.001)$, and decision regret $(r=-0.19, p=0.001)$, and positively correlated with perceived understanding $(r=0.30, p<0.001)$. Change in knowledge from baseline to time 2 was not correlated with any outcomes ( $p$ values $=0.28$ to 0.88 ).

To extend these findings with multivariate analyses, we conducted four hierarchical linear multiple regressions 


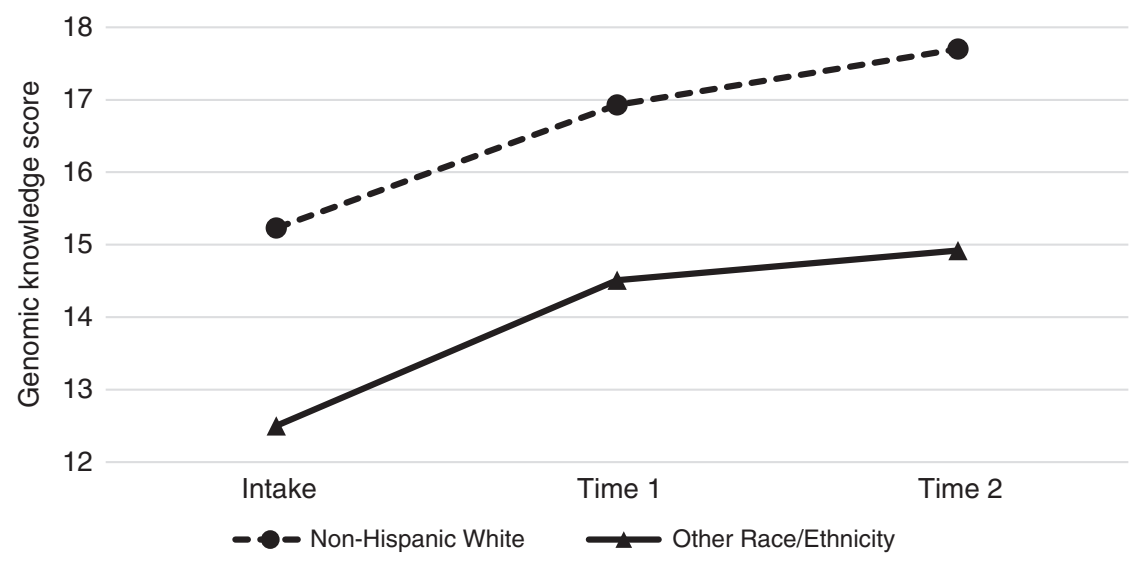

Fig. 1 Genomic knowledge scores for non-Hispanic White participants versus participants from another race/ethnic group.

Table 2 Summary of findings from hierarchical linear multiple regression analyses predicting outcomes

\begin{tabular}{|c|c|c|c|c|c|c|c|c|}
\hline \multirow[t]{2}{*}{ Variables } & \multicolumn{2}{|c|}{$\begin{array}{l}\text { Test-related } \\
\text { distress } \\
(N=313)\end{array}$} & \multicolumn{2}{|c|}{$\begin{array}{l}\text { Test-related } \\
\text { uncertainty } \\
(N=313)\end{array}$} & \multicolumn{2}{|c|}{$\begin{array}{l}\text { Decision regret } \\
(N=313)\end{array}$} & \multicolumn{2}{|c|}{$\begin{array}{l}\text { Perceived } \\
\text { understanding } \\
(N=309)\end{array}$} \\
\hline & Step $1 \beta$ & Step $2 \beta$ & Step $1 \beta$ & Step $2 \beta$ & Step $1 \beta$ & Step $2 \beta$ & Step $1 \beta$ & Step $2 \beta$ \\
\hline Adult patient participant (vs. caregiver participant) & $-0.161^{b}$ & $-0.164^{c}$ & $-0.100^{\mathrm{a}}$ & $-0.101^{\mathrm{a}}$ & $0.114^{\mathrm{b}}$ & $0.114^{b}$ & $-0.130^{b}$ & $-0.132^{b}$ \\
\hline $\begin{array}{l}\text { Assignment to decision group (vs. control group or not } \\
\text { randomized) }\end{array}$ & -0.02 & -0.02 & - & - & - & - & - & - \\
\hline Diagnostic result uncertain (vs. negative) & $0.168^{c}$ & $0.169^{c}$ & $0.220^{d}$ & $0.222^{d}$ & - & - & - & - \\
\hline Non-Hispanic White (vs. other race/ethnicity) & - & - & -0.066 & -0.053 & -0.052 & -0.038 & $0.103^{a}$ & 0.056 \\
\hline Household income & - & - & $-0.110^{\mathrm{a}}$ & $-0.100^{\mathrm{a}}$ & -0.044 & -0.034 & $0.157^{c}$ & $0.125^{b}$ \\
\hline High school or less education (vs. more education) & - & - & 0.060 & 0.041 & 0.071 & 0.051 & -0.051 & 0.015 \\
\hline Functional health literacy (vs. lower health literacy) & - & - & $-0.199^{d}$ & $-0.183^{c}$ & -0.069 & -0.053 & $0.117^{b}$ & 0.059 \\
\hline
\end{tabular}

(Table 2). Findings offered limited support for hypothesis 3. Greater baseline genomic knowledge was associated with lower test-related distress $(p=0.008)$ and greater perceived understanding $(p<0.001)$, but not with test-related uncertainty $(p=0.34)$ or decision regret $(p=0.32)$. Greater positive change in genomic knowledge was not associated with testrelated distress $(p=0.11)$, test-related uncertainty $(p=0.42)$, or decision regret $(p=0.39)$, but it was marginally positive associated with perceived understanding $(p=0.07)$.

\section{DISCUSSION}

In this study of adult patients and caregivers of pediatric patients undergoing diagnostic exome sequencing in the NCGENES study, we found that general genomic knowledge increased across study assessments; that social resources that could help people acquire, comprehend, and retain genomic knowledge were associated with gaps in baseline genomic knowledge; and that those gaps persisted throughout the study despite printed educational materials and in-person counseling by clinicians with specialized genetics training. Additionally, analyses revealed associations between baseline general genomic knowledge and several psychological outcomes. These findings extend understanding of genomic knowledge in diagnostic genome-scale sequencing in a way that can guide additional research and interventions.

The greatest increase in general genomic knowledge occurred from enrollment, prior to which participants received a mailed educational brochure, to several weeks after an enrollment visit at which participants met with a genetic counselor. This increase was maintained until after 
return of results, when participants again met with study genetics experts. The effect size of this change was just under one-half a standard deviation, falling into a range that makes it likely to be clinically significant. ${ }^{31}$

Multivariate analyses revealed higher genomic knowledge at baseline for non-Hispanic White participants and participants with higher income, education, and health literacy compared with participants of other races and ethnicities and those with lower income, education, and health literacy. Although all of these subgroups demonstrated gains in knowledge during the study, the gaps apparent at baseline persisted throughout the study, despite our educational efforts. On one hand, these general gains are encouraging given our goal of helping participants understand sequencing and its findings. Yet, they also underscore the need to investigate specific strategies for addressing subgroup gaps in genomic knowledge. In addition to the general knowledge studied here, research is needed to investigate the role of personal, contextualized knowledge.

We believe that simply offering more education and counseling is not sufficient for achieving the goal of "functional" genomics knowledge (e.g., ref. ${ }^{17}$ ). Researchers should investigate evidence-based methods for presenting information to enhance learning (e.g., changing the design of documents and adding pictures and/or video-based education). ${ }^{32}$ Additionally, research on patient-centered communication, informed decision making, and shared decision making suggests that features of clinician communication (e.g., how they exchange information, manage uncertainty, respond to patient emotions, and explore patient preferences and values) may influence patients' and caregivers' subjective response to clinical encounters, the extent to which they develop a shared understanding of information covered and its implications, and their acquisition and retention of information. ${ }^{33}$ Training can teach clinicians relevant patient-centered skills and enhance their cultural competence. $^{34}$

The importance of baseline genomic knowledge in our study also underscores a need to improve genomic literacy at the population level ${ }^{35}$ to help ensure that people suddenly faced with sequencing are prepared for it. Hurle and colleagues $^{35}$ discussed reasons people have difficulty understanding genomic information, including its inherent complexity as well as inadequate knowledge of basic biology and mathematics, limited linguistic skills, and difficulties understanding risk-related concepts such as absolute versus relative risk and population versus individual risk. Addressing these issues is likely to require a multipronged approach, including broad interventions such as $\mathrm{K}-12$ education and mediafocused interventions. These researchers also called for involving community stakeholders to ensure that interventions are culturally and linguistically appropriate and that they are implemented in ways that maximize their reach. We agree. We believe these types of efforts would provide people with a framework for understanding genomic concepts that they may encounter in clinical encounters, ${ }^{36}$ enhancing people's preparation for understanding and applying information from their own sequencing in collaboration with clinicians who provide expert, patient-specific guidance (e.g., accounting for specific findings, family and personal health history, and other risk factors).

The need for action to improve genomic knowledge is bolstered by our findings regarding genomic knowledge and its associations with several psychological sequencing outcomes. Greater baseline genomic knowledge was associated with lower test-related distress and greater perceived understanding of diagnostic exome sequencing after return of results, consistent with the assumption that general genomic knowledge can provide a foundation for better psychological sequencing outcomes. ${ }^{1-4,7,8,37}$ Contrary to expectations, genomic knowledge was not associated with lower decision regret. Agreeing to sequencing may be a relatively low stakes decision in the setting of undiagnosed disease, unlikely to generate regret. Indeed, our study clinicians observed that participants did not seem to struggle with this decision. Potential for regret may also be reduced if those at highest risk declined to join the study, or by the perception that any information yielded by sequencing is valuable, even if results are negative. ${ }^{38}$ Participants also may have felt their clinicians would continue to search for information, so even negative or uncertain results could change in the future. Furthermore, aspects of informed decision making not evaluated in this study (e.g., values concordance ${ }^{1}$ ) may affect decision regret.

Likewise, the lack of an association between general genomic knowledge and test-related uncertainty was unexpected; however, knowledge may be most likely to reduce uncertainty when it is not general genomic knowledge, as measured in this study, but rather personalized knowledge that is specific to patients' unique health condition, personal and family history, and sequencing findings. It may be useful to conduct research to clarify how each type of knowledge is uniquely or jointly associated with psychological sequencing outcomes.

We did not find that change in knowledge was associated with the psychological outcomes we studied. It may be that the changes we observed were too modest to improve these outcomes, or that information that is more personally relevant and concrete would have a greater impact. In NCGENES, study geneticists and genetic counselors provided participants with both general genomic knowledge and contextualized genomic information personalized specifically to their or their child's condition and findings. Psychological research suggests that personalized information may be easier to learn and that it could have a greater impact on psychological responses because it is more personally relevant and useful ${ }^{39}$-it applies directly to participants' own personal and family health situation. Developing a way to measure acquisition and understanding of personalized genomic knowledge (e.g., knowledge specific to patients' genomic findings and to their and their family's health history and future risks) would enable investigation of research questions such as whether, compared with general knowledge, personalized knowledge has a greater emotional impact and is more easily 
remembered, or whether general genomic knowledge provides a foundation for better understanding of personalized knowledge.

We note several limitations of this study. First, study education was not specifically designed to address the topics on our genomic knowledge scale. Thus, observed increases in knowledge may underrepresent increases possible with more targeted educational approaches. Additionally, some participants may have read our educational brochure before completing the baseline genomic knowledge measure, possibly overestimating baseline knowledge and underestimating change in knowledge. Participants may have varied systematically in whether they read the educational brochure. Second, we did not measure participant understanding and retention of personalized knowledge they received in the study. Future research on the interplay between general genomic knowledge and personalized, contextualized knowledge could inform education and counseling efforts. Third, we did not consider participant age in analyses due to missing caregiver age data. Because age is correlated with other variables (e.g., education, income), it is difficult to know whether this could have affected our findings. Fourth, we adapted the MICRA measure to refer to genomics and for interview administration, with unknown effects on its validity. Finally, future research should include larger samples of specific minority or underserved subgroups to advance research on challenges they face.

Strengths of the current study include its longitudinal study design, large sample size, and inclusion of patients with diverse medical conditions, which may enhance the generalizability of study findings. Also, knowledge of genetic causes of disease is an aspect of health literacy that is increasingly important to understand given growing availability of genetic and genomic testing. Higher genetic/genomic knowledge may impact acceptance of this technology. ${ }^{40}$ Our findings show that it can also influence people's potential for negative experiences associated with diagnostic exome sequencing, and that important gaps can persist despite educational efforts.

\section{SUPPLEMENTARY INFORMATION}

The online version of this article (https://doi.org/10.1038/s41436019-0600-4) contains supplementary material, which is available to authorized users.

\section{ACKNOWLEDGEMENTS}

This research was supported by the National Human Genome Research Institute of the National Institutes of Health under award number U01HG006487 (principal investigators: James P. Evans, Jonathan S. Berg, Karen E. Weck, Kirk C. Wilhelmsen, and Gail E. Henderson). The content is solely the responsibility of the authors and does not necessarily represent the official views of the National Institutes of Health. The study sponsor was not involved in study design, data collection, analysis or interpretation of data, writing this report, or the decision to submit the report for publication.

\section{DISCLOSURE}

The authors declare no conflicts of interest.

Publisher's note: Springer Nature remains neutral with regard to jurisdictional claims in published maps and institutional affiliations.

\section{REFERENCES}

1. Bowen DJ, Allen JD, Vu T, Johnson RE, Fryer-Edwards K, Hart A Jr. Theoretical foundations for interventions designed to promote informed decision making for cancer screening. Ann Behav Med. 2006;32:202-210.

2. Institute of Medicine (US) Committee on Quality of Health Care in America. Crossing the quality chasm: a new health system for the 21st century. Washington, DC: National Academies Press; 2001.

3. Jefford M, Moore R. Improvement of informed consent and the quality of consent documents. Lancet Oncol. 2008;9:485-493.

4. Makoul G, Clayman ML. An integrative model of shared decision making in medical encounters. Patient Educ Couns. 2006;60:301-312.

5. Biesecker BB, Schwartz MD, Marteau TM. Enhancing informed choice to undergo health screening: a systematic review. Am J Health Behav. 2013;37:351-359.

6. Marteau TM, Dormandy E, Michie S. A measure of informed choice. Health Expect. 2001;4:99-108.

7. Haga SB, Barry WT, Mills R, et al. Public knowledge of and attitudes toward genetics and genetic testing. Genet Test Mol Biomarkers. 2013;17:327-335.

8. Lea DH, Kaphingst KA, Bowen D, Lipkus I, Hadley DW. Communicating genetic and genomic information: health literacy and numeracy considerations. Public Health Genomics. 2011;14:279-289.

9. Foreman AK, Lee K, Evans JP. The NCGENES project: exploring the new world of genome sequencing. NC Med J. 2013;74:500-504.

10. Roche MI, Griesemer I, Khan CM, et al. Factors influencing NCGENES research participants' requests for nonmedically actionable secondary findings. Genet Med. 2019;21:1092-1099.

11. Secretary's Advisory Committee on Genetics, Health, and Society. Genetics education and training. Washington, DC: Department of Health \& Human Services; 2011.

12. Lautenbach DM, Christensen KD, Sparks JA, Green RC. Communicating genetic risk information for common disorders in the era of genomic medicine. Annu Rev Genomics Hum Genet. 2013;14:491-513.

13. Smerecnik CM, Mesters I, de Vries NK, de Vries H. Educating the general public about multifactorial genetic disease: applying a theory-based framework to understand current public knowledge. Genet Med. 2008; 10:251-258.

14. Langer MM, Roche MI, Brewer NT, et al. Development and validation of a genomic knowledge scale to advance informed decision making research in genomic sequencing. MDM Policy Pract 2017;2.

15. Kaphingst KA, Blanchard M, Milam L, Pokharel M, Elrick A, Goodman MS. Relationships between health literacy and genomics-related knowledge, self-efficacy, perceived importance, and communication in a medically underserved population. J Health Commun. 2016;21 suppl 1:58-68.

16. Schmidlen TJ, Scheinfeldt L, Zhaoyang R, et al. Genetic knowledge among participants in the Coriell personalized medicine collaborative. J Genet Couns. 2016;25:385-394.

17. Baker DW. Reading between the lines: deciphering the connections between literacy and health. J Gen Intern Med. 1999;14:315-317.

18. Hurtado-de-Mendoza A, Graves K, Gomez-Trillos S, et al. Provider's perceptions of barriers and facilitators for Latinas to participate in genetic cancer risk assessment for hereditary breast and ovarian cancer. Healthcare (Basel). 2018;6:E116. pii

19. Krakow M, Ratcliff CL, Hesse BW, Greenberg-Worisek AJ. Assessing genetic literacy awareness and knowledge gaps in the US population: results from the Health Information National Trends Survey. Public Health Genomics. 2017;20:343-348.

20. Fitzgerald-Butt SM, Klima J, Kelleher K, Chisolm D, McBride KL. Genetic knowledge and attitudes of parents of children with congenital heart defects. Am J Med Genet A. 2014;164A:3069-3075.

21. Molster C, Charles T, Samanek A, O'Leary P. Australian study on public knowledge of human genetics and health. Public Health Genomics. 2009;12:84-91. 
22. Donovan KA, Tucker DC. Knowledge about genetic risk for breast cancer and perceptions of genetic testing in a sociodemographically diverse sample. J Behav Med. 2000;23:15-36.

23. Hughes C, Gomez-Caminero A, Benkendorf J, et al. Ethnic differences in knowledge and attitudes about BRCA1 testing in women at increased risk. Patient Educ Couns. 1997;32:51-62.

24. Singer E, Antonucci T, Van Hoewyk J. Racial and ethnic variations in knowledge and attitudes about genetic testing. Genet Test. 2004;8:31-43.

25. Haga SB, Barry WT, Mills R, et al. Public knowledge of and attitudes toward genetics and genetic testing. Genet Test Mol Biomarkers. 2013:17:327-335.

26. Rini C, Khan CM, Moore E, et al. The who, what, and why of research participants' intentions to request a broad range of secondary findings in a diagnostic genomic sequencing study. Genet Med. 2018;20:760-769.

27. Davis TC, Crouch MA, Long SW, et al. Rapid assessment of literacy levels of adult primary care patients. Fam Med. 1991;23:433-435.

28. Félix-Ortiz M, Newcomb MD, Myers $\mathrm{H}$. A multidimensional measure of cultural identity for Latino and Latina adolescents. Hisp J Behav Sci. 1994;16:99-115.

29. Cella D, Hughes C, Peterman A, et al. A brief assessment of concerns associated with genetic testing for cancer: the Multidimensional Impact of Cancer Risk Assessment (MICRA) questionnaire. Health Psychol. 2002;21:564-572.

30. Morris SB, DeShon RP. Combining effect size estimates in meta-analysis with repeated measures and independent-groups designs. Psychol Methods. 2002;7:105-125.

31. Sloan JA, Cella D, Hays RD. Clinical significance of patient-reported questionnaire data. J Clin Epidemiol. 2005;58:1217-1219.
32. Sheridan SL, Halpern DJ, Viera AJ, Berkman ND, Donahue KE, Crotty K. Interventions for individuals with low health literacy: a systematic review. J Health Commun. 2011;16(suppl 3):30-54.

33. Street RL Jr., Makoul G, Arora NK, Epstein RM. How does communication heal? Pathways linking clinician-patient communication to health outcomes. Patient Educ Couns. 2009;74:295-301.

34. Renzaho AM, Romios P, Crock C, Sonderlund AL. The effectiveness of cultural competence programs in ethnic minority patient-centered health care - a systematic review of the literature. Int J Qual Health Care. 2013;25:261-269.

35. Hurle B, Citrin T, Jenkins JF, et al. What does it mean to be genomically literate?: National Human Genome Research Institute Meeting Report. Genet Med. 2013;15:658-663.

36. Bernhardt BA, Roche MI, Perry DL, Scollon SR, Tomlinson AN, Skinner D. Experiences with obtaining informed consent for genomic sequencing. Am J Med Genet A. 2015;167A:2635-2646.

37. Chapman R, Likhanov M, Selita F, Zakharov I, Smith-Woolley E, Kovas $Y$. New literacy challenge for the twenty-first century: genetic knowledge is poor even among well educated. J Community Genet. 2018; 10:73-84

38. Skinner $D$, Raspberry KA, King $M$. The nuanced negative: meanings of a negative diagnostic result in clinical exome sequencing. Sociol Health IIIn. 2016:38:1303-1317.

39. Priniski SJ, Hecht CA, Harackiewicz JM. Making learning personally meaningful: a new framework for relevance research. J Exp Educ. 2018:86:11-29.

40. Ashida S, Goodman M, Pandya C, et al. Age differences in genetic knowledge, health literacy and causal beliefs for health conditions. Public Health Genomics. 2011;14:307-316. 